\title{
Eine erfreuliche Normalität. Konversationelle Praktiken des Normalisierens und Emotionalisierens bei der Befundmitteilung in der HIV-Sprechstunde
}

\begin{abstract}
Ausgehend von Überlegungen zur Bedeutung von Normalität und Emotionalität in medizinischen Gesprächen wende ich mich im vorliegenden Beitrag zwei Praktiken der Mitteilung und Bewertung HIV-spezifischer Blutwerte in ärztlichen Sprechstunden mit HIV-positiven Patient/innen zu: Während beim Emotionalisieren das Vorliegen unauffälliger Befunde als Ereignis herausragend positiver Valenz behandelt wird, stellen normalisierende Evaluierungen den Therapieerfolg als erwartbar dar. Der Einsatz normalisierender und/oder emotionalisierender Evaluierungspraktiken ist folgenreich für den weiteren sequenziellen Verlauf der Befundmitteilung. Ihre kombinierte Verwendung spiegelt ein z. T. paradoxes Bild von HIV und seiner Behandelbarkeit wider, das durch ein Spannungsverhältnis zwischen Exzeptionalität und Normalität geprägt ist.
\end{abstract}

Keywords: HIV, Arzt/Patient-Gespräche, medizinische Gesprächsforschung, Befundmitteilungen, Normalisieren, Emotionen in der Interaktion

\section{Einleitung: Normalität und Emotionalität im Gespräch}

\subsection{Normalität und Krankheit}

Normal und Mitglieder der Wortgruppe (Normalität, Norm, Normativität) ${ }^{1}$ weisen in ihrer alltagsinteraktionalen Verwendungsweise Ambiguitäten und Überlappungsbereiche auf. Als wissenschaftliche Begrifflichkeit eingesetzt, muss Normalität bereichsspezifisch definiert werden (Horstmann 2016: 7). Bührig

1 Siehe Bührig (2009) zur historischen Entwicklung der Wortgruppe um das Adjektiv normal; zur Etymologie der Begriffe Normalität und Normativität siehe Horstmann (2016).

Alexandra Groß, Universität Bayreuth, Germanistische Linguistik, alexandra.gross@unibayreuth.de

Ә Open Access. (c) 2021 Alexandra Groß, publiziert von De Gruyter. (c) BY-NC-ND Dieses Werk ist lizenziert unter einer Creative Commons Namensnennung - Nicht-kommerziell - Keine Bearbeitung 4.0 International Lizenz. https://doi.org/10.1515/9783110688696-020 
(2009) führt an, dass Normalität eine qualitative sowie eine quantitative Achse aufweist: „Qualitativ bezeichnet ,Normalität‘ die einer Norm entsprechende Eigenschaft oder Beschaffenheit; quantitativ betrachtet bezeichnet ,Normalität‘ die Verteilung von Typen bzw. ausgewählter Merkmale im Mittelbereich einer Normalverteilung“ (Bührig 2009: 52). Auf Erfahrung oder auf wissenschaftlichen Erkenntnissen basierende Normen gelten in alltäglichen Kontexten zumindest implizit nicht nur als übliche oder frequenteste Merkmalsausprägung, sondern als Bewertungsgrundlage. Karl Pearson, der die Bezeichnung „Normalkurve“ für die Gaußsche Normalverteilung geprägt hat, weist selbst darauf hin, dass die Bezeichnung dazu führe, niedrig frequente Merkmalsausprägungen als unnormal wahrzunehmen (Horstmann 2016). Huber (2011: 42) stellt entsprechend fest: „,S]cientific standards, so-called 'norms of reference' (Referenzwerte), might also be intertwined with ethical and/or societal normativity to a significant degree“ und betont, dass diese Interpretation auf einem Missverständnis beruhe.

Ethnovorstellungen von Normalität sind also aufgeladen mit Soll-Vorstellungen menschlichen Seins und Handelns. Das Normale entpuppt sich hier sowohl als Vergleichs- als auch als Zielkategorie und dient als Orientierungspunkt für menschliches Handeln (Bührig unter Verweis auf eine Arbeit von Sohn (1999)). Es ruft zugleich die Kategorie des Abweichenden auf. Dabei kann das, was als normal gilt, ganz unterschiedlich bewertet werden: Normative Implikationen von Normalität können sowohl ,als Mittelmaß-Plädoyer wie auch als Ablehnung einer Mittelmaß-Orientierung und dann als Verbesserungsstreben“ auftreten (Horstmann 2016: 8).

Die eine Möglichkeit der präskriptiven Deutung von normal $=$ Homöostase $=$ gut bzw. unnormal $=$ Abweichung $=$ schlecht wird im Rahmen der westlichen Biomedizin evident: Das Krankheitsgeschehen wird als Funktionsstörung physischer Prozesse gesehen, Gesundheit dagegen als anzustrebender Zustand. Beides ist ebenfalls das Ergebnis normativer Setzungen (Horstmann 2016). Zugleich existiere auch auf rein deskriptiver Ebene eine „quite fussy notion, that is to say 'loose concept' of normal functioning in medicine“ (Huber 2011: 44), nämlich als reine Negation, als Nicht-Vorliegen von etwas Krankhaftem. ${ }^{2}$ Zudem nimmt auch die Krankheit selbst in der Medizin den Stellenwert des Normalen ein (Horstmann 2016), da nur ein Patient, der seine Beschwerden als doctorable (Heritage \& Robinson 2006) legitimieren kann, in den medizinischen Betrieb eintreten und Nutznießer therapeutischer Behandlungen werden kann.

2 Zur theoretischen Darlegung des Normalitäts- und des Krankheitsbegriffs in der modernen Medizin siehe z. B. Hucklenbroich (2008) und Horstmann (2016). 
Aus Patientensicht wird der krankhafte Zustand durch das Auftreten von Beschwerden und die Diagnosestellung Realität. Die psychologisch relevante Erfahrung von Kontinuität in der eigenen Biographie zur Sicherung der eigenen Identität (z. B. Straub 2000) oder - phänomenologisch gesehen - die Erwartung des „und-so-weiter“ im Alltäglichen (Schütz \& Luckmann 2003: 88) erweisen sich durch das Auftreten einer Erkrankung als bedroht.

Zwei medizinische Besonderheiten von HIV im Vergleich zu anderen Krankheitsgeschehen seien an dieser Stelle genannt: Zum einen bleibt HIV in den meisten Fällen über Jahre hinweg asymptomatisch. Bei nicht wenigen Patient/ innen ist die Feststellung von HIV daher ein unerwarteter Zufallsbefund und in seiner Asymptomatizität phänomenologisch nicht mit ihrem scheinbar gesunden Körpergefühl zusammenzubringen. Entsprechend fragt ein US-amerikanischer HIV-Patient in einem der untersuchten Gespräche: „Do I really have this?“ (HIV-Korpus). Patient/innen erfahren bei der Diagnose von HIV, dass sie sich vom medizinischen Standpunkt gesehen in einem Zustand der Nicht-Normalität befinden. Mit der Diagnose HIV ist die subjektiv wahrgenommene Normalität als Scheinnormalität enttarnt; eine HIV-bedingte Verschlechterung des Gesundheitszustands bis hin zum Schreckensbild Aids hängt nun wie ein Damoklesschwert über den Patient/innen.

Eine zweite Besonderheit von HIV ist seine Unheilbarkeit. Das Virus kann zwar durch die sog. hochaktive antiretrovirale Kombinationstherapie (abgekürzt: ART) über viele Jahre hinweg in Schach gehalten werden; die Anzahl der Viruskopien im Blut (=Viruslast) bleibt bei gutem Therapieansprechen unter der Nachweisgrenze. Jedoch erfordert diese Wiederherstellung eines infektiologisch negativ-normalen Zustands eine stete patientenseitige Compliance sowie eine regelmäßige medizinische Überwachung. Die Therapie eliminiert HIV nicht, sie stellt den Normalzustand also nicht wieder her, sondern erschafft eine wie auch in Zeiten der Corona-Pandemie durch politische und medizinische Akteur/innen oft zitierte - neue Normalität, die fragil und abhängig von ihrer steten medikamentösen Herstellung ist. Eine neue Normalität mutet zunächst als Paradoxon an, da sie die alte Normalität als kontrastierende Vergleichsfolie impliziert und damit eben auf etwas (noch) nicht Normales verweist. Die neue Normalität von HIV-Patient/innen beinhaltet zudem, dass die Medikation Nebenwirkungen verursachen kann. Die aus medizinischer Sicht wieder Normwerte aufweisenden Patientenkörper fühlen sich unter Umständen also ganz und gar nicht normal an, paradoxerweise aufgrund der therapeutischen Bemühungen, einen quasi-normalen Zustand wiederherzustellen.

Eine weitere, zeitliche Dimension von Normalität kommt in der HIV-Therapie (wie auch bei anderen Krankheitsgeschehen) zum Tragen: die Vorstellung eines üblichen zeitlichen Verlaufs einer Erkrankung und eines Therapieanspre- 
chens. Auch um diese Entwicklungsachse von Normalität wird es im Folgenden gehen: um ein typisches Verlaufsmuster also innerhalb etwas Unnormalem wie einer HIV-Infektion, das innerhalb eines pathologischen Zustands als anstrebenswert gilt und das - qua Verweis auf die gute medizinische Behandelbarkeit - als erwartbar behandelt wird. Dabei ist das Wiederherstellen eines normalen Gesundheitszustands das Ziel der Therapie. Dies wird häufig explizit mit Berufung auf Normalität benannt, nämlich: Patient/innen eine (annähernd) normale Lebenserwartung zu ermöglichen. Auch medizinhistorisch gesehen hat HIV eine Entwicklung von einer ausgesprochen bedrohlichen hin $\mathrm{zu}$ einer normalen - das heißt: behandelbaren - Infektion durchgemacht. Während die dem alten AIDS zugeordnete Phase des Exzeptionalismus durch eine regelrechte Inszenierung der Erkrankung geprägt ist (Jann 2019), tritt mit der Erfindung der antiretroviralen Kombinationstherapie Mitte der 1990er Jahre eine Normalisierung (Wright \& Rosenbrock 2012) ein, die das neue AIDS (besser: das neue HIV) einläutet. Diskursiv persistiere aber weiterhin der Eindruck, „dass es sich um ein medizinisches und soziales Phänomen handelt, das besonderer Aufmerksamkeit bedarf“ (Jann 2019: 83). Die Analyse entsprechender konversationeller Praktiken kann neben der Untersuchung des öffentlich-medialen Diskurses Aufschluss darüber geben, welche Deutungsmuster im Umgang mit HIV bestehen.

\subsection{Normalität als konversationelle Herstellungsleistung}

Aus einer ethnomethodologischen Perspektive ist Normalität nicht als gesetzter Zustand, sondern als fortwährende interaktive Herstellungsleistung zu begreifen (Garfinkel 1967; Sacks 1984). Dabei können Gesprächsgegenstände (wie z. B. erzählte Ereignisse), Emotionen des Gegenübers sowie auch das Gesprächshandeln selbst zum Gegenstand normalisierender Bemühungen der Interagierenden werden und auf allen sprachlichen Ebenen sichtbar werden. Die sprachlich-interaktive Herstellungsleistung von Normalität ist immer das Ergebnis von Aushandlungsprozessen.

Sacks (1984) beschreibt das Bestreben, im Gespräch Normalität beizubehalten bzw. herzustellen; er benennt dieses als doing being ordinary. Teil dessen sei z. B., bei der Beschreibung einer Person nicht die Form ihrer Augenbrauen hervorzuheben. Solche konversationellen Normen verwiesen auf Wissen, was in einer sozialen Gemeinschaft als normal gelte und wie man sich in Gesprächen entsprechend verhalte. Sacks beobachtet, dass Personen auch bei der Schilderung außergewöhnlicher oder bedrohlicher Ereignisse den Eindruck von “nothing happened” (Sacks 1984: 419) beibehalten und so ihre „ordinariness“ hervorheben (Sacks 1984: 414). 
Solche Bemühungen sind auch für Arzt/Patient-Gespräche - genauer: für bedrohliche Diagnosemitteilungen - belegt. Die Interagierenden betreiben angesichts eines solchen interaktiven und psychologischen Bruchs konversationellen Aufwand, um diesen bereits antizipativ abzumildern und ins Stocken geratene Gesprächsroutinen möglichst rasch wiederherzustellen. Maynard (v. a. 2003) zeigt, wie Diagnosen schwerwiegender Erkrankungen zunächst häsitativ angebahnt (forecasting, Maynard 1996) und modalisiert vorgebracht werden. Z. T. binden Ärzt/innen die Patient/innen in einen sequenziell aufwändigen Übermittlungsprozess (perspective display series) mit ein (Maynard 1992). Günthner (2017: 39) beschreibt für Diagnosegespräche in der Onkologie als „projektive Praktik“ der Diagnosemitteilung, dass Ärzt/innen bekannte Elemente der Vorgeschichte (z. B. vorhergehende diagnostische Vermutungen) aufgreifen und diese als gemeinsamen Wissensbestand darstellen. Die Diagnose selbst wird so als etwas präsentiert, das bereits im Raum stand. Weiterhin verweilen Ärzt/innen nicht lange bei Bedeutungsklärung und Empathiebekundungen (siehe Abschnitt 1.3), sondern gehen rasch zur Therapieplanung über.

Die identifizierten Verfahren dienen zum einen dazu, eine schwerwiegende Diagnose nicht $\mathrm{zu}$ abrupt in die patientenseitige Normalität einbrechen $\mathrm{zu}$ lassen, und zum anderen, die Patient/innen in ein „es geht weiter“ zurückzuholen. Sie sind damit sprachlich-interaktive Praktiken des Normalisierens.

Diese sind in medizinischen Gesprächen auch in weniger schwerwiegenden Kontexten belegt: Bührig (2009: 55) beschreibt für prä-operative Aufklärungsgespräche, dass sich Mediziner/innen auch hier an Normalität orientieren, indem der Eingriff nebst möglichen Komplikationen „unter die Normalität des medizinischen Alltags“ subsumiert werden.

Auch das Thematisieren von Emotionen im medizinischen Gespräch selbst kann Bezugspunkt normalisierender konversationeller Verfahren werden, wie u. a. Heritage \& Lindström (2012) zeigen: In einem Nachsorgegespräch zwischen einer Hebamme und einer jungen Mutter wird das Ausbleiben überschwänglicher Muttergefühle durch die Hebamme auf eine normale Entwicklung der Mutter-Kind-Bindung zurückgeführt. Die Studie demonstriert in besonderer Weise, dass konversationelle Verfahren des Normalisierens zugleich das Regulieren von Emotionen bedeuten: Sie haben das Ziel, durch Perspektivänderungen auf die Bedeutung eines Sachverhalts X-in Heritage \& Lindströms Studie eben eine Emotion selbst - die Emotionen des anderen neu auszurichten. Ihr Einsatz demonstriert dabei institutionelle Erklärungsmacht: Auch drastische Schilderungen seitens der Mutter bezüglich ihrer Distanzierung von ihrem Baby werden durch die Hebamme konversationell in den Bereich des Normalen hineingenommen.

Die genannten Forschungsarbeiten zeigen, wie medizinische Akteur/innen sich angesichts existenzieller, plötzlich hereinbrechender oder auch nur antizi- 
pierter Bedrohungen der patientenseitigen Normalität bemühen, diese im Gespräch wiederherzustellen bzw. eine entsprechende Perspektive auf medizinische Gegebenheiten auszurichten.

\subsection{Manifestation und Regulation von Emotionen in medizinischen Gesprächen}

„Displays of emotion and affect are a central part of everyday actions and social relations“ (Sorjonen \& Peräkylä 2012: 3), sie erweisen sich als sensitiv gegenüber Interaktionskontext und konversationellen Erfordernissen. „Trägerinnen“ emotionaler Haltungen oder entsprechender Rezipientenreaktionen können emotionsaffine konversationelle Handlungen wie z. B. Bewertungen (z. B. Goodwin \& Goodwin 1992) und Empathiebekundungen (Kupetz 2015) sein; weiterhin werden evaluativ-emotionale Haltungen als second layer konversationeller Handlungen und Aktivitäten evident. Das Display von Affiliation als empathisches Reagieren auf Sprecheremotionen ist z. B. in Alltagsnarrativen essenzieller Teil einer adäquaten Rezipienz und hat Einfluss auf die anschließenden Sprecheraktivitäten (z. B. Peräkylä et al. 2015).

Ärztliche Gespräche scheinen hinsichtlich des Umgangs mit Emotionen insofern von informellen Gesprächstypen abzuweichen, als erstere als sachorientiert auf die Behandlung somatischer Beschwerden gelten (Fiehler 2005). Patient/ innen orientieren sich an diesen institutionellen Erfordernissen und Beschränkungen, indem sie üblicherweise von der Schilderung emotionaler Erlebnisse absehen und ihr Krankheitserleben im Gespräch mit dem behandelnden Arzt fragmentieren (Lalouschek 1995). Institutionelle Sachlichkeit und Lösungsorientierung in Gesprächen mit medizinischen Professionellen zeigen sich z. B. dann, wenn Ärzt/innen auf das Äußern belastender Gefühle mit dem Anbieten von Medikamentengaben reagieren. Ruusuvuori (2007) stellt fest, dass medizinische Akteur/innen zwar mit patientenseitigen Gefühlsäußerungen affiliieren, allerdings v. a. mit dem Ziel, rasch zur Lösung des medizinischen Problems überzugehen.

Trotz aller Sach- und Lösungsorientierung macht „der Umgang mit eigenen, aber auch mit fremden Emotionen“ einen Teil des ärztlichen und des patientenseitigen Gesprächshandelns aus (Lindemann 2015: 155). Emotionen erscheinen auf der Gesprächsoberfläche, wenn sie durch Verbalisierungen oder (para)verbale Ausdrucksressourcen manifestiert werden oder das Gesprächshandeln von Patient/innen als emotional gedeutet wird (Fiehler 2005; Imo 2017). Dass das Thematisieren, das (para)verbale Anzeigen, das Einfühlen, aber auch das Regulieren von Emotionen sogar ein zentraler Handlungsaspekt sein kann, zeigt Günthner 
(2017) mit ihrer Untersuchung onkologischer Diagnosegespräche: Von Seiten der Ärzt/innen wird hier emotionale Anteilnahme auf unterschiedlichen sprachlich-interaktiven Ebenen (gedämpfte Stimmqualität, verstehensdokumentierende Äußerungen) signalisiert. Weiterhin wird das patientenseitige Gesprächshandeln nach der Diagnosemitteilung (v. a. Verstummen) als emotional gedeutet. Imo (2017) stellt den Aufforderungscharakter von Emotionsdarstellungen heraus: Sie initiieren Trostpraktiken wie die „das Schlechte ist X, aber das Gute ist Y“-Strategie (Imo 2017: 215), die als ein Verfahren der Emotionsregulation vorhandene oder antizipierte Patientenemotionen modulieren und eine hoffnungsvolle Perspektive etablieren sollen. Maynard et al. (2015) beschreiben als weitere ärztliche Strategie die Umdeutung onkologischer Befunde: Gleichbleibend große Tumoren werden als lobenswertes Ereignis vorgeschlagen. Das Ausbleiben einer Schrumpfung des Tumors als antizipierte patientenseitige Sicht soll auf diese Weise blockiert werden.

Die Analysen in Abschnitt 3 werden in ähnlicher Weise zeigen, dass das sprachlich-interaktive Emotionsmanagement in der HIV-Sprechstunde darauf abzielt, positive Emotionen zu erzeugen, indem unauffällige Befunde als herausragendes Ereignis bewertet werden. Zudem wird sich herausstellen, dass Mediziner/innen mittels Wahl einer emotionalisierenden und/oder normalisierenden Evaluationspraktik die Befundmitteilung insofern lenken können, als diese weitergeführt und auch durch Patient/innen emotional beantwortet wird oder zu einem Ende kommen soll.

\section{Befundmitteilungen in der HIV- Routinesprechstunde: informieren und evaluieren}

\subsection{Hintergrundinformationen zur HIV-Therapie und zum vorliegenden Korpus}

Die Therapie einer HIV-Infektion bedeutet die lebenslange Einnahme von Medikamenten und regelmäßige Kontrolltermine z. B. in der HIV-Schwerpunktpraxis. Hier werden sowohl die Entwicklung des HIV-Status des/der Patient/in als auch die Entwicklung HIV-unspezifischer physiologischer Funktionen überprüft. Ersterer wird anhand der Viruslast und der Anzahl der CD4-Helferzellen bestimmt. Diese beiden HIV-spezifischen Blutparameter gelten als die relevanten Indikatoren für die Wirksamkeit der medikamentösen Langzeittherapie: Der medizinisch 
erstrebenswerte Fall ist, dass die Viruslast dauerhaft niedrig und das Immunsystem stabil bleibt. Relevante Parameter des unspezifischen Blutbilds ( $u$. a. Leberund Nierenwerte) zeigen an, dass der/die HIV-Patient/in nicht unter erkennbaren Nebenwirkungen der ARV-Medikation leidet und keine HIV-bedingten Erkrankungen aufweist. Das Gespräch mit dem/der behandelnden Mediziner/in ist das zentrale Interaktionsereignis im Rahmen der HIV-Therapie.

Die vorliegende Untersuchung wurde in einer großen auf HIV spezialisierten Ambulanz in einem Universitätsklinikum in Deutschland durchgeführt. Die Datenerhebung fand im Rahmen eines ethnographischen Forschungsaufenthalts in der Einrichtung statt. Datengrundlage sind 71 zumeist dyadische Gespräche zwischen HIV-positiven Patient/innen und HIV-Spezialist/innen. Diese wurden audiotechnisch aufgezeichnet, mittels GAT2 (Selting et al. 2009) transkribiert und mit Methoden der linguistischen Gesprächsanalyse (z. B. Deppermann 2008) analysiert. Eine ausführliche Untersuchung der Gespräche findet sich bei Groß (2018).

Das Setting der untersuchten Infektionsambulanz sieht vor, dass die Patient/ innen in einem Wartebereich auf das Aufrufen durch den Arzt/die Ärztin warten. Die Interagierenden tauschen also i.d. R. bereits Grußformeln aus, bevor sie das Sprechzimmer betreten. In ihrem Verlauf weisen die Gespräche - neben der Eröffnung und Beendigung des medizinischen Kerngesprächs - folgende obligatorische, vorwiegend instrumentelle Aktivitäten auf: Befundmitteilung (siehe Abschnitt 2.2), Blutdruck messen und Körpergewicht bestimmen. Da HIV-Patient/innen die Sprechstunde unabhängig von ihrem Wohlbefinden in regelmäßigen Abständen aufsuchen und die Kontrolle des HIV-Status im Gespräch zumeist im Vordergrund steht, können die untersuchten HIV-Sprechstunden als Routinekontrollgespräche klassifiziert werden. Dennoch werden auch akute Beschwerden bearbeitet, falls solche vorliegen. In diesem Fall werden die Beschwerden exploriert und diagnostisch interpretiert; weiterhin werden Therapieentscheidungen getroffen und/oder Überweisungen ausgesprochen.

\subsection{Die Aktivität der Befundmitteilung}

Entsprechend der allgemeinen Relevanz des Blutbilds, welches das Gelingen oder Misslingen der antiretroviralen Therapie anzeigt, stellt die Aktivität der Befundmitteilung den kommunikativen Kern der untersuchten HIV-Sprechstunden dar. Neben informierenden Gesprächshandlungen (die Mitteilung der Werte) ist sie durch Evaluationen geprägt. Der folgende Ausschnitt verdeutlicht die sequenzielle Organisation sowie die Rekurrenz evaluierender Handlungen: 
Ausschnitt 1: Übermittlung und Evaluierung der Blutwerte (HIV-Korpus, R2-2806AP, 352-371 Sek.)

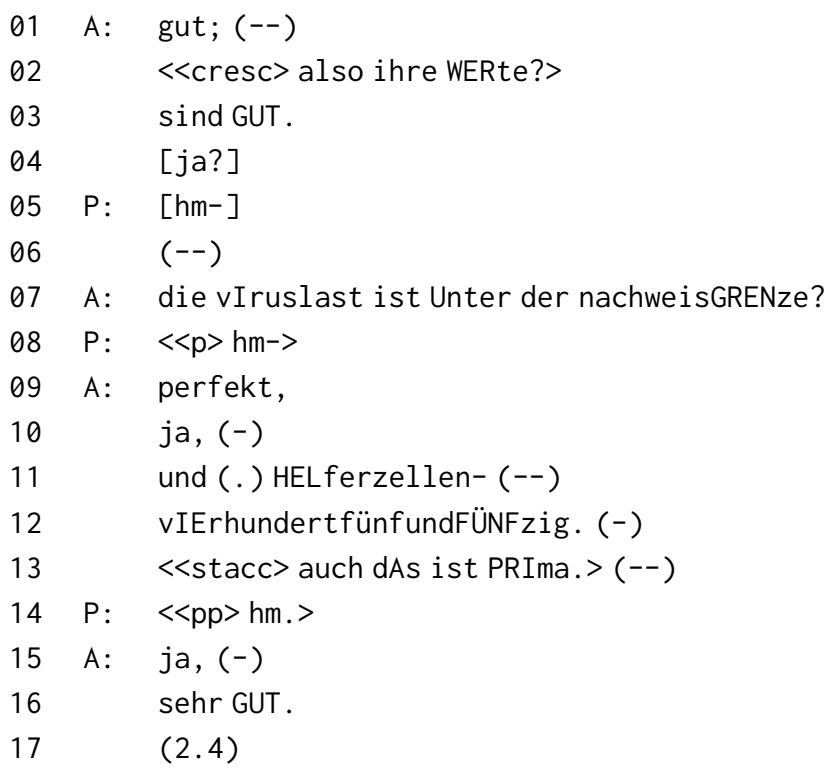

Mit der Gliederungspartikel gut initiiert der Arzt den Übergang zur Befundmitteilung (Z. 01). Er evaluiert zunächst global die Bluttestergebnisse (Z. 02-03) und informiert dann mit der Routineformel die vIruslast ist Unter der nachweisGRENze (Z. 07) über den Befund.

Die verbale Aktivität des Patienten beschränkt sich auf minimale Rezipientensignale (Z. 05, 08, 14), was zunächst unmittelbar mit der Funktion der Aktivität zusammenhängt: der Übermittlung von Befundwissen, das nur dem Mediziner mit Einsicht in die Patientenakte zugänglich ist. Dass der Patient kaum auf den Neuigkeitswert der Mitteilungen reagiert, weist bereits darauf hin, dass die gute Nachricht des unauffälligen Befundbilds nicht als Neuigkeit behandelt wird: In den untersuchten Gesprächen stellt das Vorliegen unauffälliger HIV-spezifischer Werte mit Abstand den frequentesten Fall dar; ihre Mitteilung erfolgt als konversationell präferierte Handlung auf direkte Weise und wird auch durch die Patient/innen als Normalfall behandelt. Die hieraus auch resultierende Nichtvermittlung von Wissen (etwa in ärztlichen accounts über mögliche Gründe der vorliegenden Befunde) ist ebenfalls ein Merkmal der (impliziten) Mitteilung guter Nachrichten. Letztere sichern die Fortführung des Gesprächs als Routinekontrollgespräch und erfüllen - aus psychologischer Perspektive die Erwartung von Kontinuität und Normalität. 
In den untersuchten Gesprächen zeigt sich weiterhin eine starke Routinehaftigkeit der sprachlich-interaktiven Realisierung der Aktivität: Über die Formulierungsroutinen auf der Redezugsebene hinaus (etwa die Routineformel: unter der Nachweisgrenze sein) weist die sequenzielle Organisation der Aktivität schematische Züge auf; sie ist über das Korpus hinweg fast ausschließlich durch ärztliche initiative Mitteilungen und Evaluierungen und patientenseitig-responsiv vorwiegend durch minimale Rückmelde- und Ko-Evaluierungshandlungen geprägt.

\subsection{Verfahren des Normalisierens und des Emotionalisierens in Evaluationen}

Die Evaluationen innerhalb der Aktivität der Befundmitteilung sind ein zentraler Anknüpfungspunkt für konversationelle Verfahren des Normalisierens und des Emotionalisierens, die - wie in Abschnitt 3 vertieft werden soll $-\mathfrak{u}$. a. auf lexikalischer Ebene realisiert werden: In Ausschnitt 1 evaluiert der Arzt die Werte mehrfach positiv (Z. 03: gut, Z. 13: prima) bis hochgestuft positiv (Z. 09: perfekt, Z. 16: sehr gut). Im Korpus sind weiterhin stark positive Adjektive wie wunderbar, hervorragend, oder super sowie Ausdrücke wie völlig in Ordnung belegt. Dass positive Evaluierungen von beiden Interagierenden als dazugehörige Komponente der Aktivität behandelt werden, zeigen eigenständige Bewertungen seitens der Patient/innen. Patient/innen demonstrieren hiermit auch eigene Evaluierungskompetenz und fachmedizinisches Wissen.

Die Obligatorik der Evaluationen - die wie in Ausschnitt 1 häufig mehrfach geäußert werden - mutet bei vielen Patient/innen zunächst überflüssig an angesichts dessen, dass sie die immergleichen Werte nach z. T. jahr(zehnt)elanger Behandlung einzuordnen wissen. Dennoch oder gerade deswegen reflektiert ihre Persistenz, dass sie einen essenziellen Teil des professionellen Handelns der Ärzt/innen ausmachen und aus dem reinen Ablesen von Zahlwerten die professionelle Tätigkeit der Übermittlung von Befundwissen machen (Lindström \& Mondada 2009). Die Konstruktion diagnostischer Bedeutung ist auf diese Weise auch eine Demonstration medizinischer Expertise (siehe hierzu Groß 2018).

Das zugrundeliegende interaktive Problem der Befundmitteilung ist zum einen, Intersubjektivität über die Bedeutung der Werte herzustellen, und zum zweiten, sich der Kontinuität des Behandlungserfolgs rückzuversichern. Beides wird vor allem durch die Befundevaluationen geleistet. Der gute medizinische Verlauf kann dabei etwas sein, was als freudiges Ereignis dargestellt wird (Emotionalisieren), oder aber etwas, das als erwartbar und damit als normal bewertet wird (Normalisieren). Wie die folgenden Ausschnitte zeigen, werden beide Verfahren auch kombiniert eingesetzt, wodurch der erfreuliche Therapieerfolg zu- 
gleich als erwartbar dargestellt wird. Die Wissensvermittlung qua Mitteilung und Bedeutungsklärung der HIV-spezifischen Bluttestergebnisse wird also emotional aufgeladen und kann entsprechend auf dieser Ebene reguliert werden.

\section{Der kombinierte Einsatz des Emotionalisierens und des Normalisierens bei der Befundmitteilung}

In diesem Abschnitt werden die konversationellen Praktiken des Emotionalisierens und des Normalisierens in der Aktivität der Befundmitteilung betrachtet, indem die hierfür verwendeten sprachlichen und gesprächsorganisatorischen Ressourcen identifiziert werden. Für diesen Beitrag habe ich Gesprächsausschnitte ausgewählt, in denen die Verfahren kombiniert werden, indem sie entweder sequenziell aufeinanderfolgen (Ausschnitt 2) oder sprachlich ineinandergreifen (Ausschnitt 3). Beides ist in den untersuchten Gesprächen des HIV-Korpus auch in weiteren Gesprächen belegt.

Das konversationelle Verfahren des Emotionalisierens in der Befundmitteilung bedeutet das Einnehmen eines emotional stance zum Vorliegen unauffälliger HIV-spezifischer Blutwerte, das auf diese Weise mit einer positiven Emotionalität aufgeladen und als herausragend erfreuliches Ereignis konstruiert wird.

Sehr deutlich emotionalisierend wirkt eine prosodisch-expressive Evaluierungspraktik, die im folgenden Ausschnitt 2 bereits in einer Mitteilungsäußerung beginnt (Z. 09): Die extra-starke Akzentuierung auf der ersten Silbe des Zahlworts hebt sie prosodisch hervor, hinzu kommen eine gepresste Stimmqualität bei hoher Tonlage, eine auffällig ausladend steigende Tonhöhenbewegung auf der ersten Silbe der Intonationsphrase sowie eine erhöhte Sprechlautstärke.

Ausschnitt 2: Sukzessive Verwendung von Emotionalisieren und Normalisieren (HIV-Korpus, A-1907-AP, 333-349 Sek.)

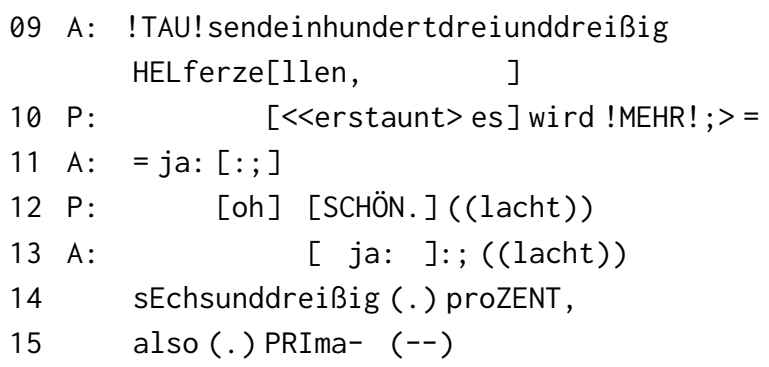


und $(-) h^{\circ}(-)$ vIrus wie immer unter der NACHweisgrenze. $=$

$17=$ das is (--) kaum mehr erWäHnenswert;

18 ja, =

$19=$ das [is: ] eh (.) STANdard.

$20 \mathrm{P}: \quad[<<\mathrm{p}>\mathrm{hm}->]$

Die Patientin greift den Display positiver Emotionen in Überlappung auf (Z. 10), affiliiert prosodisch mittels extra starker Akzentuierung und hohem pitch auf mehr, und expliziert auch mittels Lachen die positive emotionale Bedeutung des Werts. Verbal nimmt die Patientin einen evaluative stance ein, indem sie auf das Ansteigen Bezug nimmt (Z. 10), und setzt hier einen eigenen - intraindividuellvergleichenden - Bewertungsschwerpunkt.

Das gedehnte ja (Z. 11) seitens des Arztes, das mit einem hohen onset und langsam abfallendem pitch (parallel zur Realisierung von mehr durch die Patientin) realisiert wird, affiliiert wiederum mit dem patientenseitigen Display von Freude und bestätigt zugleich inhaltlich die vergleichende Einschätzung. Das beidseitige Emotionalisieren kulminiert im emphatisch intonierten Exklamativ (Z. 12), der die positive Bedeutung lexikalisch expliziert, in der hiermit überlappenden affiliierenden Bestätigung durch den Arzt (Z. 13) sowie im anschließenden Lachen beider Interagierenden.

Die Konstruktion der gemeinsamen Freude über die hohe Anzahl der Helferzellen wird also schrittweise mittels Einsatzes (para)verbaler Verfahren des Emotionalisierens etabliert: Initiativ weist bereits die Mitteilungsäußerung emotionales Potential auf, auf die responsiv ein Affiliieren bei der Ko-Evaluation erfolgt. Das ärztliche Handeln bedeutet bis hier nicht, die Werte selbst aus medizinischer Sicht als unauffällig zu bewerten, vielmehr wird das Vorliegen einer unauffälligen Helferzellenanzahl als herausragend lobenswertes, freudiges Ereignis behandelt. Dies sehe ich als empathische Praktik an, da hier nicht eine medizinisch sachorientierte Perspektive auf die Befundbedeutung, sondern eine patientenseitig emotionale Sicht vorgeschlagen (und aufgegriffen) wird.

Im weiteren Verlauf von Ausschnitt 2 werden weitere HIV-spezifische Blutwerte unter Verwendung normalisierender Praktiken mitgeteilt und evaluiert. Der Arzt teilt den prozentualen Anteil der CD4-Helferzellen an der Gesamtimmunabwehr mit (Z. 14) und bewertet ihn, eingeleitet mit dem Explikationsmarker also, als prima (Z. 15). Diese sowie die nachfolgenden Intonationsphrasen sind im Unterschied zu den vorausgehenden - prosodisch unmarkiert realisiert. Dann wird die Viruslast mit der Routineformel unter der Nachweisgrenze sein mitgeteilt (Z. 16). Das Temporaladverbial wie immer kontextualisiert, dass das Vorliegen der nicht nachweisbaren Anzahl der Viruskopien im Blut erwartbar ist, seine 
Mitteilung also keinen Neuigkeitswert hat und ergo kaum mehr erwähnenswert (Z. 17) und Standard (Z. 19) ist. Die Häsitationspraktiken (Pausen, Häsitationsmarker $e h$ ) in den Evaluierungsäußerungen weisen zusammen mit dem Telegrammstil (Z. 16) auf eine herabgesetzte konversationelle Aufmerksamkeit hin und kontextualisieren die Planung nachfolgender Aktivitäten. Auch hierdurch wird das Vorliegen der unauffälligen Viruslast normalisiert.

Im folgenden Ausschnitt 3 treten Verfahren des Emotionalisierens und des Normalisierens ebenfalls kombiniert auf, hier greifen die beiden Evaluierungspraktiken aber stärker ineinander.

Ausschnitt 3: Ineinandergreifen von Emotionalisieren und Normalisieren (HIVKorpus, H1-1706-AP, 96-118 Sek.)

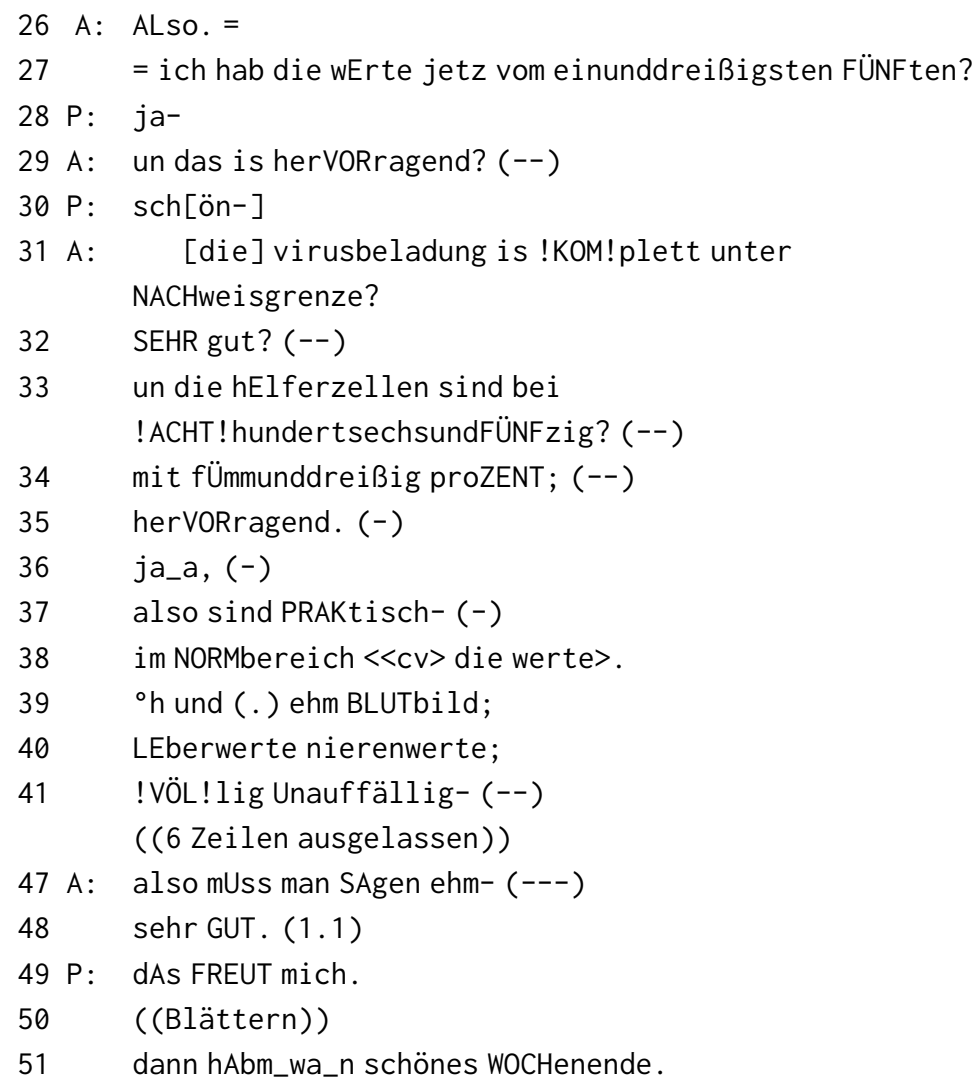

In diesem Ausschnitt werden im Unterschied zu Ausschnitt 2 direkt explizite Evaluationen der vorliegenden Werte geäußert: Mittels hochgestuft-positivem Adjektiv 
hervorragend (Z. 29) wird zunächst eine Bewertung realisiert, die vorausweisend auf alle nachfolgenden Werte referiert. Zusätzlich zeigen sich die oben beschriebenen prosodischen Praktiken des emotional stance-taking in den Mitteilungsäußerungen (Z. 31 und 33) und in weiteren Evaluierungen (Z. 32, 35): Extra-starke Akzentuierungen, eine hohe Tonhöhenvariabilität und schnelle Sprechgeschwindigkeit setzen eine weitere Handlungsnuance auf die Bewertungsaktivität; diese prosodische Gestaltung vermittelt einen Eindruck aufgeregt-affektiver Beteiligung und verwandelt die Befundmitteilung in ein emotionalisiertes Ereignis.

Im Unterschied zur Patientin in Ausschnitt 2 affiliiert der Patient hier (zumindest auf (para)verbaler Ebene) nicht: Nach einer einzigen alignierenden Evaluation (Z. 30) auf die einleitende Bewertung erfolgen weder hörbare affektive Reaktionen noch Verstehensdokumentationen, was durch den Arzt als absent behandelt wird (Z. 36). Daraufhin bewertet der Arzt, eingeleitet mit dem Reformulierungsmarker also, die Werte als praktisch im Normbereich liegend. Neben der nun unmarkierten Prosodie fungiert die Referenz auf den Normbereich als normalisierendes Verfahren. Praktisch impliziert dabei allerdings, dass die Befunde vielleicht nicht ganz an die Durchschnittswerte einer gesunden Population heranreichen und macht hierdurch zugleich eine Diskrepanz zum Normalen auf. Obgleich im Folgenden auch die Nieren- und Leberwerte als unauffällig bewertet werden (Z. 40-41), wird diese Befundnormalität zugleich mittels Intensifikator völlig hochgestuft (siehe auch Z. 31: komplett unter Nachweisgrenze). Das Zusammenspiel von einerseits prosodisch und lexikalisch realisiertem Hinweis auf Außergewöhnlichkeit und andererseits dem Hinweis auf die Übereinstimmung der unauffälligen Befunde mit Normwerten wird als Antwort auf eine zusammenfassende und damit sequenzbeendigende ärztliche Bewertung (Z. 47-48) in der Patientenäußerung (ab Z. 49) eminent: Diese drückt äußerungssemantisch Freude und eine optimistische Zukunftsprojektion aus, ist zugleich aber prosodisch unmarkiert und feststellend-neutral gestaltet.

\section{Zusammenfassung und Diskussion}

Aus den untersuchten Gesprächen lassen sich die Praktiken der Befundevaluation des Normalisierens und des Emotionalisierens auf verschiedenen sprachlich-interaktiven Ebenen (lexikalische und syntaktische Redezuggestaltung, Prosodie, sequenzielle Dynamik) beschreiben. $\mathrm{Zu}$ ihrer kombinierten Verwendung in den untersuchten Ausschnitten 2 und 3 ist Folgendes festzuhalten:

- Emotionalisieren bedeutet das Einnehmen eines emotional stance zum Vorkommen unauffälliger Blutwerte, das auf diese Weise als herausragend po- 
sitives Ereignis konstruiert wird. Ärztliche Praktiken des Emotionalisierens sind in initiativen evaluierenden Äußerungen im Rahmen der Befundmitteilung häufig belegt und können bereits im Mitteilungsbeitrag beginnen. Es werden auf prosodischer Ebene extra starke Akzentuierungen, eine hohe pitch-Variabilität sowie erhöhte Sprechlautstärke und -geschwindigkeit sowie gemeinsames Lachen (Ausschnitt 2) realisiert. Auf lexikalischer Ebene sind hochgestuft-positive Bewertungen wie sehr gut, hervorragend sowie patientenseitig Ausdrücke der Freude (das freut mich, schön) zu verzeichnen. Die Reaktionen der Patient/innen unterscheiden sich zwischen den untersuchten Ausschnitten fundamental: Während die Patientin in Ausschnitt 2 eine Art emotionale formulation äußert, indem sie die Bedeutung der ärztlichen Mitteilung expliziert, prosodisch affiliiert und Freude zeigt, reagiert der Patient in Ausschnitt 3 nicht hörbar auf die hochgestuft-positiven Evaluationen der guten Befundnachrichten, was als absent behandelt wird. Auf gesprächsorganisatorischer Ebene bewirken emotionalisierende ärztlich-initiative Befundevaluationen also eine Fortführung der Sequenz.

- Der Bezugspunkt ärztlicher Praktiken des Normalisierens sind ebenfalls Evaluierungsäußerungen. In der Bewertung wird entweder eine intraindividuelle Vergleichsperspektive im Sinne der Erwartbarkeit guter Werte bei dem/der jeweiligen Patient/in aufgemacht (Ausschnitt 2) oder es wird interindividuell auf (implizit bleibende) Normbereiche der gesunden Population verwiesen (Ausschnitt 3). Die Praktiken des Normalisierens enthalten auf lexikalischer und phrasaler Ebene das Adjektiv normal bzw. semantisch verwandte Begriffe wie unauffällig, die nominalen Ausdrücke im Normbereich und Standard und kaum mehr erwähnenswert. Sie leisten eine Konstruktion von Erwartbarkeit im Sinne von Alltäglichkeit und Routiniertheit, aber auch von Normalität im interindividuellen Vergleich. Normalisierend wirkt zudem der syntaktische Auflistungsstil der Mitteilungsäußerungen, der nicht nur gute Nachrichten qua Direktheit ihrer Mitteilung kontextualisiert, sondern auch einen reduzierten konversationellen Aufwand spiegelt. Hinsichtlich seiner prosodischen Gestaltung speist sich das Normalisieren in den untersuchten Ausschnitten aus dem Kontrast zu vorausgehenden, prosodisch markierten Äußerungen emotionalisierender Befundbewertungen. Responsiv affiliieren die Patient/innen mit den normalisierenden Mitteilungen häufig mittels minimaler Reaktionen. Sie tragen hiermit dazu bei, dass die Sequenz zu einem Ende kommen kann. Im Unterschied zu emotionalisierenden ärztlichen Evaluationen, auf die affiliierende Responsive seitens der Patient/innen gefordert sind, bringen normalisierende Bewertungsäußerungen die Sequenz (und hier auch die Mitteilungsaktivität) also eher zum Abschluss. Die beschriebenen Praktiken des Normalisierens können als professionell-distanziertes Einnehmen einer epistemischen Haltung 
$\mathrm{zu}$ einer implizit bleibenden (intraindividuellen oder interindividuellen) Norm perspektiviert werden.

- In beiden Ausschnitten folgen innerhalb der Aktivität der Befundmitteilung auf emotionalisierende normalisierende Praktiken der Befundevaluation. Die Verfahren können entweder sukzessive aufeinander folgen (Ausschnitt 2) oder/und auf verschiedenen sprachlichen Ebenen ineinandergreifen (Ausschnitt 3): In Ausschnitt 3 werden die Befunde lexikalisch und prosodisch als unauffällig eingestuft, diese Unauffälligkeit aber zugleich mittels intensivierender Ausdrücke als herausragend bewertet: komplett unter Nachweisgrenze (Z. 31), völlig unauffällig (Z. 41).

Bisherige Arbeiten zu Emotionalität in medizinischen Gesprächen haben gezeigt, dass konversationelle Verfahren des Normalisierens eingesetzt werden, um angesichts negativer Emotionen Perspektiven der Kontinuität aufzuzeigen und die Progressivität des Gesprächshandelns aufrecht zu erhalten. Im Unterschied zu konversationellen Verfahren der Emotionsregulation und des Normalisierens zur Bearbeitung negativer Emotionen sollen in der HIV-Befundmitteilung mittels kombinierten Einsatzes emotionalisierender und normalisierender Praktiken der Befundevaluation positive Emotionen erzeugt und eine medizinisch-kontrollierbare Perspektive auf gute Befundnachrichten vorgeschlagen werden.

Die untersuchten Praktiken dienen dazu, Intersubjektivität über die Bedeutung der Befunde herzustellen, auch im Sinne einer gegenseitigen Rückversicherung über den Therapieerfolg. Emotionalisieren bedeutet das Einnehmen eines emotional stance zum Vorkommen unauffälliger Blutwerte, das auf diese Weise als herausragend erfreuliches Ereignis konstruiert wird. Obwohl es durch Ärzt/innen sequenzinitiativ eingesetzt wird, sehe ich das Emotionalisieren als Praktik eines Empathie-Displays an, da hier v. a. eine antizipierte Patientensicht auf gute Befundnachrichten vorgeschlagen wird. Das Verfahren fungiert als Elizitator einer affiliierenden Reaktion seitens des Patienten.

Das konversationelle Verfahren des Normalisierens ist als Einnehmen einer distanziert-professionellen Haltung zu einem impliziten interindividuellen Vergleich bzw. zu einem intraindividuell-retrospektiven Vergleich zu werten. Eine Normalisierung findet hier auch dadurch statt, dass Patient/innen responsiv nicht mit Überraschung auf die good news, sondern nur minimal reagieren und so die Sequenzbeendigung einleiten. Normalisierende Evaluationen signalisieren den Patient/innen besonders deutlich Kontinuität und Sicherheit und schaffen eine „entsprechende Neudefinition des >anders Normalen<“ (Weingart, 2002: 38). Angesichts des Vorliegens unauffälliger Blutwerte verweisen solche Praktiken zudem ex negativo darauf, dass dies einmal ein herausragend positives Ereignis 
war; dies ist an den persistierenden, häufig hochgestuft-positiven Bewertungen seitens der Ärzt/innen zu erkennen.

Während emotionalisierende Evaluationen das Vorliegen unauffälliger Befunde als herausragend lobenswertes Ereignis darstellen und sie aus der Normalität „herauskatapultieren“, sind normalisierende Evaluationen zusammen mit minimalen Patientenreaktionen und der routinierten „Abarbeitung“ der Aktivität (Routineformeln, sequenzielle Gleichförmigkeit) Verfahren, um „,the event's ordinariness, its usualness“ (Sacks 1984: 414) hervorzuheben (siehe auch Groß 2018). Mit ihrer inhaltlichen Ausrichtung auf objektiviertes medizinisches Wissen spiegeln sie besonders explizit den institutionellen Rahmen, demonstrieren zudem medizinische Expertise und schaffen institutionelle Sachlichkeit. In Kombination verwendet, ko-konstruieren die beschriebenen Evaluierungspraktiken eine erfreuliche Normalität; sie reflektieren damit das wichtigste Ziel der HIV-Therapie: den Patient/innen „ein normales Leben“ zu ermöglichen.

Die sequenzielle Abfolge von Emotionalisieren und Normalisieren in den gezeigten Ausschnitten spiegelt auch den Behandlungsprozess von HIV wider, in dessen üblichem Verlauf die Befundmitteilung ehemals herausragende Neuigkeiten bereithielt, an die man sich zunehmend gewöhnt, wie auch HIV in seiner Geschichte „zuvor für außerordentlich - [...] gut oder böse, bedrohlich oder bereichernd - gehalten wurde, diesen Status verliert und in Wahrnehmung wie Handeln in die Welt des Bekannten, Gewohnten zurückkehrt“ (Rosenbrock \& Schaeffer 2003: 7). Dass diese neue Normalität fragil ist und einer steten Herstellung bedarf, zeigt der Einsatz der beschriebenen konversationellen Verfahren. Im Gegensatz zu einer alten Normalität, die nicht erwähnt wird, da sie nicht auffällt, muss die neue Normalität explizit als nicht mehr erwähnenswert bezeichnet werden. Sie ist damit paradoxerweise exzeptionell.

Das Aufeinanderfolgen und Ineinandergreifen der beschriebenen konversationellen Praktiken im sozialen Mikrokosmos eines ärztlichen Gesprächs spiegelt auch - wie in Abschnitt 1.1 beschrieben - die parallele Existenz von Deutungsmustern vordergründiger medizinischer Normalität und einer persistierenden Exzeptionalität von HIV. Ob Ärzt/innen in ihrem konversationellen Handeln sensibel auf Letztere reagieren, Patient/innen „dort abholen“ und in den Bereich medizinischer Normalität hereinzuholen versuchen, oder ob entsprechende diskursive Deutungsmuster im Gespräch selbst ihren Ursprung haben, mag dahingestellt sein. Die interaktive Ordnung, die hierdurch gefestigt wird, ist aber eine, in der Ärzt/innen empathisch-affektiv die Patientenperspektive antizipieren und zugleich Verlässlichkeit und professionelle Sachlichkeit demonstrieren. 


\section{Literatur}

Bührig, Kristin (2009): Zur sprachlich-interaktiven Realisierung von Normalität in der ArztPatienten-Kommunikation. In Hanneli Döhner, Olaf von dem Knesebeck \& Heidrun Kaupen-Haas (Hrsg.), Medizinsoziologie in Wissenschaft und Praxis, 51-64. Berlin: LIT. Deppermann, Arnulf (2008): Gespräche analysieren. Eine Einführung. Wiesbaden: Verlag für Sozialwissenschaften.

Fiehler, Reinhard (2005): Erleben und Emotionalität im Arzt-Patienten-Gespräch. In Mechthild Neises, Susanne Ditz \& Thomas Spranz-Fogasy (Hrsg.), Psychosomatische Gesprächsführung in der Frauenheilkunde. Ein interdisziplinärer Ansatz zur verbalen Intervention, 120-136. Stuttgart: Wissenschaftliche Verlagsgesellschaft.

Garfinkel, Harold (1967): Studies in Ethnomethodology. Englewood Cliffs/NJ: Prentice Hall. Goodwin, Charles \& Marjorie Harness Goodwin (1992): Assessments and the Construction of Context. In: Alessandro Duranti \& Charles Goodwin (Hrsg.), Rethinking Context: Language as an Interactive Phenomenon, 147-190. Cambridge: Cambridge University Press.

Groß, Alexandra (2018). Arzt/Patient-Gespräche in der HIV-Ambulanz. Facetten einer chronischen Gesprächsbeziehung. Göttingen: Verlag für Gesprächsforschung.

Günthner, Susanne (2017): Sprachliche Verfahren bei der Übermittlung schlechter Nachrichten - Sedimentierte Praktiken im Kontext onkologischer Aufklärungsgespräche. Arbeitspapiere Sprache \& Interaktion 71, 1-45.

Heritage, John \& Jeffrey Robinson (2006): Accounting for the visit: giving reasons for seeking medical care. In John Heritage \& Douglas Maynard (Hrsg.), Communication in Medical Care: Interactions between Primary Care Physicians and Patients, 48-85. Cambridge: Cambridge University Press.

Heritage, John \& Anna Lindström (2012): Knowledge, Empathy, and Emotion in a medical encounter. In Anssi Peräkylä \& Marja-Leena Sorjonen (Hrsg.), Emotion in Interaction, 256-273. New York: Oxford University Press.

Horstmann, Simone (2016): Ethik der Normalität - Zur Evolution moralischer Semantik in der Moderne (Ethik in der Praxis 39). Berlin: LIT.

Huber, Lara (2011): Norming Normality: On Scientific Fictions and Canonical Visualisations. Medicine Studies 3, 41-52.

Hucklenbroich, Peter (2015): Normal - anders - krank? Begriffsklärungen und theoretische Grundlagen zum Krankheitsbegriff. In Dominik Groß, Sabine Müller \& Jan Steinmetzer (Hrsg.), Normal - anders - krank. Akzeptanz, Stigmatisierung und Pathologisierung im Kontext der Medizin, 3-30. Berlin: MWV.

Imo, Wolfgang (2017): Trösten. Eine sprachliche Praktik in der Medizin. Muttersprache 127, 197-225.

Jann, Nina (2019): Das neue AIDS? Die Diskursivierung von HIV und AIDS im SPIEGEL von 1996-2013. In Alexandra Groß, Ramona Pech \& Ivan Vlassenko (Hrsg.), HIV/AIDS. Interdisziplinäre Perspektiven, 75-94. Berlin: LIT.

Kupetz, Maxi (2015): Empathie im Gespräch - Eine interaktionslinguistische Perspektive. Tübingen: Stauffenburg.

Lalouschek, Johanna (1995): Ärztliche Gesprächsausbildung. Eine diskursanalytische Studie zu Formen des ärztlichen Gesprächs. Opladen: Westdeutscher Verlag. 
Lindemann, Katrin (2015): Emotionen in der medizinischen Kommunikation. In: Albert Busch \& Thomas Spranz-Fogasy (Hrsg.), Handbuch Sprache in der Medizin, 154-170. Berlin, Boston: De Gruyter.

Lindström, Anna \& Lorenza Mondada (2009): Assessments in Social Interaction: Introduction to the Special Issue. Research on Language and Social Interaction 42 (4), 299-308.

Maynard, Douglas (1992): On Clinicians Co-implicating Recipients' Perspective in the Delivery of Diagnostic News. In Paul Drew \& John Heritage (Hrsg.), Talk at work, 331-358.

Cambridge: University Press.

Maynard, Douglas (1996): On „Realization“ in everyday life: The forecasting of bad news as a social relation. American Sociological Review 61, 109-131.

Maynard, Douglas (2003): Bad News, Good News - Conversational order in everyday talk and clinical settings. Chicago: The University of Chicago Press.

Maynard, Douglas, Dagoberto Cortez \& Tony C. Campbell (2015): ,End of life' conversations, appreciation sequences, and the interaction order in cancer clinics. Patient Education and Counselling 99 (1), 92-100.

Peräkylä, Anssi (2015): Sharing the Emotional Load: Recipient Affiliation Calms Down the Storyteller. Social Psychology Quarterly 78 (4), 301-323.

Rosenbrock, Rolf \& Doris Schaeffer (2003): Die Normalisierung von Aids: Erinnerungen für die Zukunft. In: Rolf Rosenbrock \& Doris Schaeffer (Hrsg.), Die Normalisierung von Aids. Politik - Prävention - Krankenversorgung (Ergebnisse sozialwissenschaftlicher AidsForschung 23), 7-8. Berlin: Edition Sigma.

Ruusuvuori, Johanna (2007): Managing affect: Integrating empathy and problem solving in two types of health care consultations. Discourse Studies 9, 597-622.

Sacks, H. (1984): On doing „being ordinary“. In J. Maxwell Atkinson \& John Heritage (Hrsg.), Structures of Social Action, 413-429. Cambridge: University Press.

Schütz, Alfred \& Thomas Luckmann (2003): Strukturen der Lebenswelt. Konstanz: UVK.

Selting, Margret, Peter Auer, Dagmar Barth-Weingarten, Jörg Bergmann, Pia Bergmann, Karin Birkner et al. (2009): Gesprächsanalytisches Transkriptionssystem 2 (GAT2). Gesprächsforschung - Online-Zeitschrift zur verbalen Interaktion 10, 353-402.

Sohn, Werner (1999): Bio-Macht und Normalisierungsgesellschaft - Versuch einer Annäherung. In Werner Sohn \& Herbert Mehrtens (Hrsg.), Normalität und Abweichung. Studien zur Theorie und Geschichte der Normalisierungsgesellschaft, 9-29. Opladen: Westdeutscher Verlag.

Sorjonen, Marja-Leena \& Anssi Peräkylä (2012): Introduction. In: Marja-Leena Sorjonen \& Anssi Peräkylä (Hrsg.), Emotion in Interaction, 3-15. New York: Oxford University Press.

Straub, Jürgen (2000): Biographische Sozialisation und narrative Kompetenz. Implikationen und Voraussetzungen lebensgeschichtlichen Denkens in der narrativen Psychologie. In: Erika M. Hoerning (Hrsg.), Biographische Sozialisation, 137-163. Stuttgart: Lucius \& Lucius.

Weingart, Brigitte (2002): Ansteckende Wörter. Repräsentationen von AIDS. Frankfurt (Main): Suhrkamp.

Wright, Michael T. \& Rolf Rosenbrock (2012): AIDS - Zur Normalisierung einer Infektionskrankheit. In: Günter Albrecht \& Axel Groenemeyer (Hrsg.), Handbuch soziale Probleme, 195-218. Wiesbaden: Springer. 
